\title{
Thermal Insensitive Optical Liquid Level Sensor based on Excessively Tilted Fibre Bragg Grating
}

\author{
Chengbo Mou, Kaiming Zhou, Lin Zhang, Ian Bennion \\ Photonics Research Group, Aston University, Birmingham, B4 7ET, UK \\ Authore-mail address: mouc@aston.ac.uk
}

\begin{abstract}
We demonstrate a liquid level sensor based on the surrounding medium refractive index (SRI) sensing using of an excessively tilted fibre Bragg grating(ETFBG). The sensor has low thermal cross sensitivity and high SRI responsivity. (C2008 Optical Society of America

OCIS codes: (060.3735) Fiber Bragg gratings; (280.4788) Optical sensing and sensors
\end{abstract}

\section{Introduction}

Liquid level sensing is of great interest in modern industry. Various ways of liquid level sensing based on mechanical[1], electrical[2], and optical[3,4] techniques have been reported. Among those, electrical sensors are widely applied. However, optical sensors can provide safe monitoring of the liquid level in conductive or explosive environment due to its intrinsic dielectric property. The reported optical fibre liquid level sensors rely on microbending of optical fibre [3], or utilise surrounding medium refractive index (SRI) sensitivity of long period fibre grating (LPG) [4]. However, LPG sensors have intrinsically high thermal cross sensitivity which is undesirable for many applications and provide better results only for liquids with SRI much higher that water based solutions. In this paper, we report a novel optical fibre liquid level sensor based on SRI sensitivity of an excessively tilted fibre Bragg grating (ETFBG).

\section{Grating fabrication}

The ETFBG used in our experiment is a Bragg grating tilted at $\sim 78^{\circ}$ which was UV-inscribed in standard single mode fibre (Corning SMF-28) using a 244nm UV source from a CW frequency doubled $\mathrm{Ar}^{+}$laser and the scanning mask technique. The fibre samples were hydrogenated under $150 \mathrm{bar}$ at an elevated temperature of $80^{\circ}$ for 48 hours prior to UV processing.

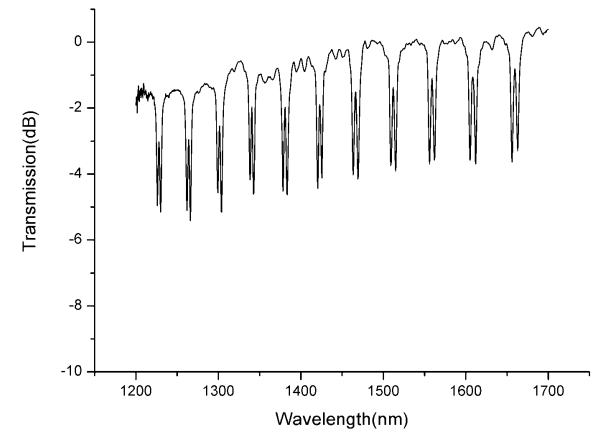

(a)

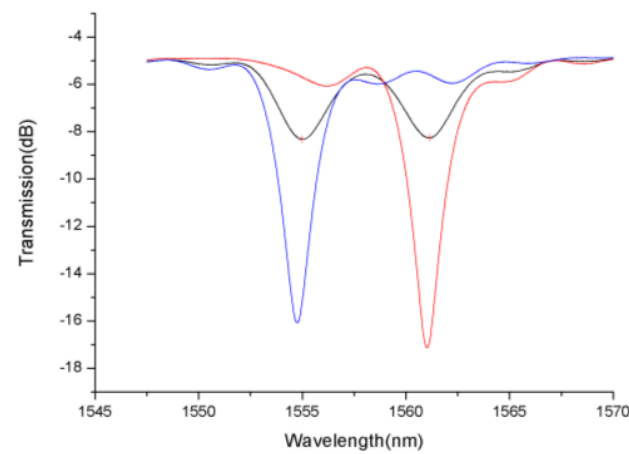

(b)

Figure.1.(a) Series of loss bands from 1200 to 1700nm measured using unpolarised light; (b) a paired peaks measured using polarised light showing a separation of $\sim 6 \mathrm{~nm}$;

A custom-designed mask of a period $6.6 \mu \mathrm{m}$ was specially purchased to ensure that grating responses can be monitored by the broadband source in $1200 \mathrm{~nm}$ to $1700 \mathrm{~nm}$ region [5]. During the UV inscription, the amplitude mask was tilted at $\sim 73^{\circ}$ to induce in-fibre fringes at $\sim 77^{\circ}$. The scanned fibre length is about $12 \mathrm{~mm}$ which is defined by the tilted angle and the length of the mask. The gratings were then annealed at $100^{\circ} \mathrm{C}$ for 3 days for stabilisation. The spectra of the gratings were examined by the use of a broadband source and an optical spectrum analyser. A polarisation scrambler was incorporated to get the full spectrum and the polarisation effect was verified by changing the polarisation state of the probing light using a polariser and polarisation controller. Fig.1.(a) and (b) show the transmission spectra of the ETFBG measured by un-polarised light for whole spectral range and polarised light just 
for one pair of the peaks. It can be seen that large tilted structure induces strong polarisation effect to the grating response, as the coupled cladding modes exhibit polarisation splitting.

\section{SRI sensitivity of ETFBG}

As the mode coupling involving high order cladding modes, ETFBGs are intrinsically sensitive to SRI change. We characterised the SRI response of an ETFBG by immersing the grating in a series of index gel with different refractive indexes, and recorded its transmission spectra.

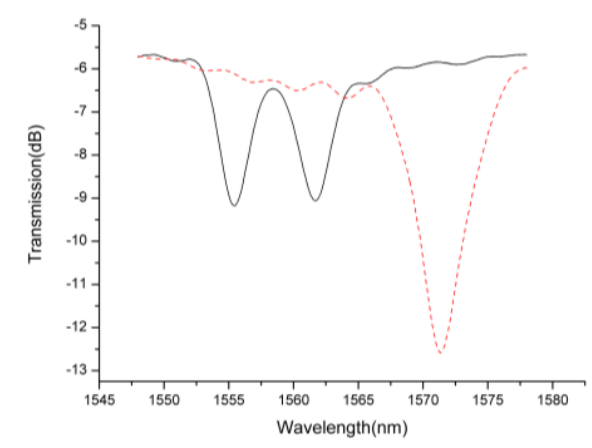

(a)

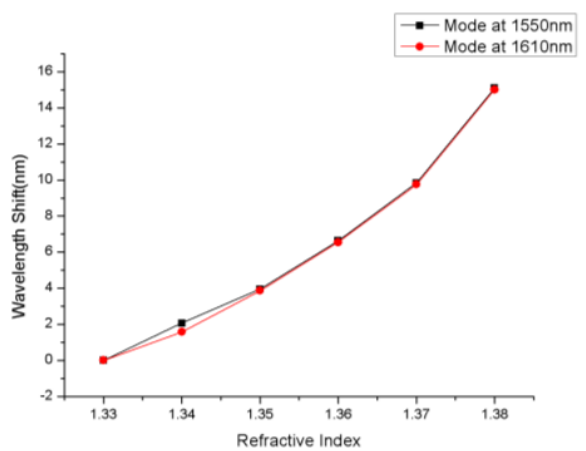

(b)

Figure 2 (a) polarisation mode splitting vanished when immersing the grating in the water accompanied with a wavelength shift, $(b) S R I-$ induced wavelength shift when index varies from 1.33 to 1.38 for two modes at $\sim 1550 \mathrm{~nm}$ and $\sim 1610 \mathrm{~nm}$

We noted that when the grating was submerged by the index matching gel, the two paired peaks evolved to one strong peak shown in Fig.2(a) and the merged single peak thus shifts with increasing SRI. Fig. 2(b) plots the wavelength shift of the two merged single peaks around $1550 \mathrm{~nm}$ and $1610 \mathrm{~nm}$ for SRI change from 1.33 to 1.38 . We can see from the figure that the shift rate is similar for both peaks and the wavelength shift reaches almost $16 \mathrm{~nm}$ for the two peaks when the SRI reaches 1.38 . We estimate that the SRI sensitivity is $\sim 320 \mathrm{~nm} / \mathrm{RIU}$ (refractive index unit) near 1.33, which is much higher than reported typical LPGs [6].

\section{Liquid level sensing experiment}

The section of the fibre containing the grating was taped to a plastic tube then immersed gradually into the water in a beaker. A slot was milled on the surface of the tube, providing full contact of the grating with water and maintaining grating straight without inducing bend loss. The beaker was mounted on a vertically micrometer driven stage to control the water level. If an ETFBG was partially immersed in the liquid, it can be regarded as two separate gratings with different surrounding media. Similar to an LPG liquid level sensor [4], for the same coupled cladding modes, the two sub gratings will generate two attenuation bands at different phase match wavelengths due to different SRIs. We have used just one polarised peak (as shown the left peak in Fig. 3) of the ETFBG around $1560 \mathrm{~nm}$ and examined its spectral evolution when it was gradually submerged into water. Fig.3 demonstrates transmission spectra measured for three different liquid levels: the left peak is under air surrounding only, the middle two peaks occur when the grating was emerged a half way to the water and the single peak on the right is under full water surrounding. When the liquid level changes we clearly see the transmission losses of the two peaks corresponding to the air- and water-surrounded gratings change, providing a mechanism to measure the water level.

In the experiment, we clearly saw that when the water level increases, the loss peak of the emerged grating grows stronger and shifts to the longer wavelength side slightly and vice versa for the air surrounded grating. The entirely water surrounded peak shifted by $12 \mathrm{~nm}$ from the peak under air. This behaviour has a totally different trend from the LPGs [4]. Fig.3 (b) shows the change of transmission losses of the two peaks corresponding to the air- and water-surrounded tilted gratings. If we just examine the water-surrounded peak we see it is not sensitive initially but gives a quite linear response region when the water level changes from $3 \mathrm{~mm}$ to $10 \mathrm{~mm}$. The air-surrounded peak is initially sensitive and the peak strength (loss) decreases with increasing water level with a slightly unsmooth linear response for the same region. 

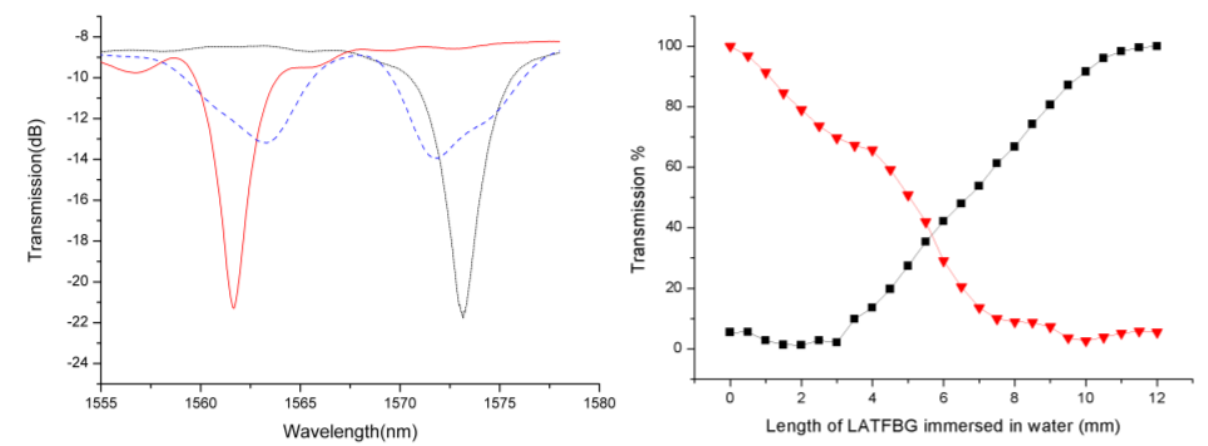

Figure 3 (a)Transmission spectra of the ETFBG for a single polarised peak around 1560nm: the left-side and righ-side single peaks and the middle dual peaks correspond to the grating surrounded by air, water and a half way into the water, respectively. (b) Plots of transmission changes measured for the water-surrounded ( $\mathbf{\nabla})$ and air-surrounded ( $\mathbf{\square})$ sub ETFBGs.

Although LPGs based liquid level sensors have been demonstrated, they have problems on temperature cross sensitivity and thus may need extra scheme for temperature compensation. It is shown in Fig.4 that from room temperature to $80^{\circ} \mathrm{C}$, there is nearly no wavelength shifts for the two paired peaks of the ETFBG, indicating this device is almost thermal-insensitive. This indicates that for liquid level detection, the use of ETFBG represents a much simpler scheme as no need for consideration of temperature compensation.

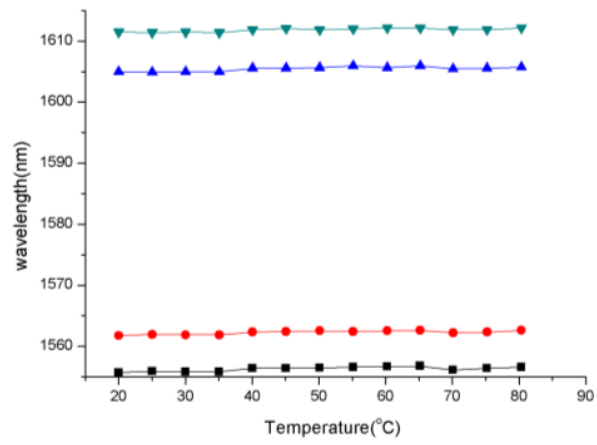

Figure.4. Wavelength shifts of two paired peaks of the ETFBG against the temperature change.

\section{Conclusion}

We have demonstrated an optical liquid level sensor based on SRI sensing of a fibre grating with excessively tilted structure. We have not just demonstrated level sensing principle using such a device but also evaluated its temperature sensitivity and found it is almost thermal-insensitive. In comparison with LPG based liquid level sensors, ETFBGs have two unique advantages as they are more sensitive to low index liquid and do not impose thermal cross-sensitivity.

\section{References}

[1] B.W.Northway, N.H.Hancock, and T.Cong-Tran, "Liquid level sensors using thin walled cylinders vibrating in circumferential modes," Meas.Sci.Technol. 6,85-93 (1995)

[2] F.N.Toch, G.C.M.Meijer, and M.Van-der-Lee, "A new capacitive precision liquid-level sensor," Proceedings of the conference on Precision Electromagnetic Measurements, (Institute of Electrical and Electronics Engineers, Piscataway, N.J., 1996) pp.356-357

[3] S.F.Knowles, B.E.Jones, S.Purdy, and C.M.France, "Multiple microbending optical-fiber sensors for measurement of fuel quantity in aircraft fuel tanks," Sens.Actuators A 68,230-323 (1998).

[4] S. Khaliq, S.W. James, and R. P. Tatam, “Fiber-optic liquid-level sensor using a long-period grating,” Opt. Lett. 26,1224-1226 (2001).

[5] Kaiming Zhou, Lin Zhang, Xianfeng Chen, and Ian Bennion,"Optic sensors of high refractive-index responsivity and low thermal cross sensitivity that use fiber Bragg gratings of >80 tilted structures," Opt.Lett. 31,1193-1195 (2006).

[6] X.Shu, B.A.L.Gwandu, Y.Liu, L.Zhang, and I.Bennion, "Sampled fiber Bragg grating for simultaneous refractive-index and temperature measurement," Opt.Lett. 17,774-776 (2001). 